\title{
Dynamo action between two rotating discs
}

\author{
A. ARSLAN† and A. J. MESTEL $*^{*}$ \\ $\dagger$ Department of Aeronautics, Imperial College London, London, UK \\ $\ddagger$ Department of Mathematics, Imperial College London, London, UK
}

(Submitted June 2020)

\begin{abstract}
Dynamo action is considered in the region between two differentially rotating infinite discs. The boundaries may be insulating, perfectly conducting or ferromagnetic. In the absence of a magnetic field, various well-known self-similar flows arise, generalising that of von Kármán. Magnetic field instabilities with the same similarity structure are sought. The kinematic eigenvalue problem is found to have growing modes for $R e_{m}>R_{c} \simeq 100$. The growth rate is real for the perfectly conducting and ferromagnetic cases, but may be complex for insulating boundaries. As $R e_{m} \rightarrow \infty$ it is shown that the dynamo can be fast or slow, depending on the flow structure. In the slow case, the growth rate is governed by a magnetic boundary layer on one of the discs. The growing field saturates in a solution to the nonlinear dynamo problem. The bifurcation is found to be subcritical and nonlinear dynamos are found for $R e_{m} \gtrsim 0.7 R_{c}$. Finally, the flux of magnetic energy to large $r$ is examined, to determine which solutions might generalise to dynamos between finite discs. It is found that the fast dynamos tend to have inward energy flux, and so are unlikely to be realised in practice. Slow dynamos with outward flux are found. It is suggested that the average rotation rate should be non-zero in practice.
\end{abstract}

Keywords: Laminar Dynamo; von Karman flow

\section{Introduction}

The generation of a magnetic field by the motion of electrically conducting fluids is important in many geophysical and astrophysical problems. An extensive review of the subject can be found in Roberts and Soward (1992). Simple analytical models, especially those which extend into the nonlinear regime, are in short supply. Recently Vaz et al. (2018) showed that in terms of cylindrical coordinates $(r, \theta, z)$, flows in an annulus of the form

$$
\boldsymbol{u}=\left(-\frac{\psi(r)}{r}, z w(r), z \frac{\psi^{\prime}(r)}{r}\right) \quad \text { in } a<r<b
$$

could generate a magnetic field with the same structure

$$
\boldsymbol{B}=\left(-\frac{\chi(r)}{r}, z C(r), z \frac{\chi^{\prime}(r)}{r}\right)
$$

The similarity in structure of the Navier-Stokes and magnetic induction equations permitted fully nonlinear solutions of that form. The governing PDEs reduce to ODEs, enabling detailed investigation.

Another class of flows with this property, this time linear in the radial rather than the axial coordinate, are of the Von Kármán (1921) type,

$$
\boldsymbol{u}=\left(-r H^{\prime}(z), r G(z), 2 H(z)\right) \quad \text { in } 0<z<1 .
$$

These describe the motion between two infinite discs at $z=0,1$ and are typically driven by differential rotation, $G(0) \neq G(1)$. These flows depend on two parameters and are known to give rise to more than

${ }^{*}$ Corresponding author. Email: j.mestel@imperial.ac.uk 
one solution branch. They have been examined by a number of authors, for example Batchelor (1951), Stewartson (1953), Hewitt and Al-Azhari (2009) and are reviewed in Zandbergen and Dijkstra (1987). In this paper we demonstrate that these flows may give rise to spontaneous growth of magnetic fields, that is, they constitute a fluid dynamo. We also determine nonlinear steady states, where the field reacts back on the driving flow.

It is not easy in the laboratory to attain a magnetic Reynolds number large enough for dynamo action, but various experiments have achieved this, such as those in Riga (Gailitis et al. 2001) and Karlsruhe (Stieglitz and Mller 2001). The geometry closest to the idealisation of this paper is the Von-KármánSodium (VKS) experiment (Nore et al. 2018, Berhanu et al. 2010, Monchaux et al. 2009), which generated a dynamo between two finite, counter-rotating discs of high magnetic permeability. The spherical geometry of Zimmerman et al. (2013) also bears some similarity to Von Kármán flow.

We shall not consider all possible dynamo modes. Rather, we investigate fields with the same spatial similarity structure as the velocity field. This reduces the problem to a set of coupled ODEs. It has the further advantage that we can readily consider nonlinear interactions of the Lorentz force with the (laminar) flow. We are therefore able to obtain solutions to the full, nonlinear dynamo problem without further assumptions. Since both velocity and magnetic fields are axisymmetric it might be expected that dynamo action would be ruled out by Cowling's theorem Cowling (1933). However this is not the case because of the unbounded domain in the radial direction. We discuss in section 5 the extent to which our results apply to finite discs in a larger container with a decaying external field. Some of the solutions involve an energy flux away from the discs, which are likely to form dynamos in the larger system. However, others involve an energy flux inwards from infinity, and should not really be considered dynamos. We nevertheless refer to all growing modes as "dynamos" in this paper. We find that exactly counter-rotating discs are not ideal for dynamo action, which has implications for the design of experiments.

We consider three types of boundary conditions on the discs, insulating, perfectly conducting and highly permeable. For the kinematic dynamo, the growth rates are shown to be real for the perfectly conducting and permeable cases, but they may be complex when the exterior is an insulator. Saturated steady solutions to the full dynamo problem are found. These persist below the critical value of $R e_{m}$ for kinematic dynamo action.

The outline of this paper is as follows. In section 2 we derive the governing equations for the nonlinear dynamo. In section 3 we investigate the kinematic dynamo problem, and seek the range of $R e_{m}$ for which magnetic growth occurs. The behaviour at large $R e_{m}$ is analysed. When the flow is away from one disc and towards the other, it is shown that the dynamo is slow, with the growth rate behaving as $\operatorname{Re}_{m}^{-1 / 3}$ as $R e_{m} \rightarrow \infty$. However, fast dynamo modes, with $\lambda \not \rightarrow 0$ can occur when the flow is directed towards each disc. This behaviour is confirmed numerically. It is demonstrated that the ferromagnetic boundaries encourage the dynamo.

In section 4 steady solutions of the fully nonlinear system are sought. Several solution branches are found. Nonlinear subcritical solutions are found for $R e_{m}<R_{c}$. When the boundaries are perfectly conducting, it is found that as $R e_{m}$ increases the nonlinear solution approaches a purely hydrodynamic flow with a constant current in the $z$-direction. For insulating boundaries, however, the nonlinear solution persists as $R e_{m} \rightarrow \infty$. In section 5 we investigate the flux of magnetic energy to large values of $r$, to determine which solutions might form genuine dynamos in a finite system. We conclude in section 6 .

\section{Governing equations}

We consider an incompressible Newtonian fluid of kinematic viscosity $\nu$ confined between two infinite discs separated by a distance $d$. We nondimensionalise lengths with respect to $d$ and work with dimensionless cylindrical polar coordinates $(r, \phi, z)$ in the domain $0<z<1$ as depicted in figure 1 . We also scale the angular speed of the disc at $z=0$ to unity, so that the rotation rate of the upper disc is $s=\omega_{2} / \omega_{1}$. The hydrodynamic Reynolds number then takes the form $R e=d^{2} \omega_{1} / \nu$. The hydrodynamic problem therefore depends on the two parameters $s$ and $R e$. 

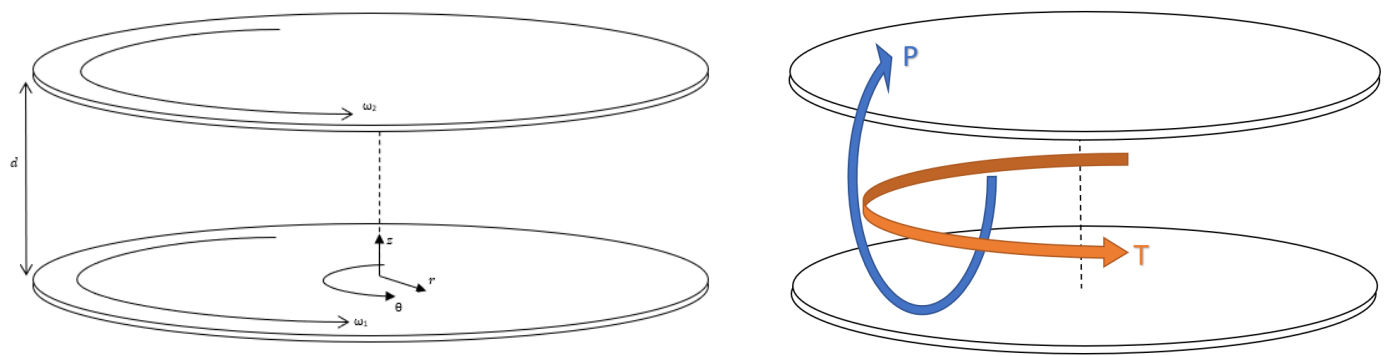

Figure 1. The geometry and field decomposition.

In kinematic dynamo theory the velocity field is regarded as given and self-excited solutions of the magnetic induction equations are sought (Roberts and Soward 1992). In non-dimensional form, using scales $d$ and $\omega_{1}^{-1}$ for length and time respectively, these are given by

$$
\begin{aligned}
\boldsymbol{\nabla} \cdot \boldsymbol{B} & =0 \\
\frac{\partial \boldsymbol{B}}{\partial t} & =\boldsymbol{\nabla} \times(\boldsymbol{u} \times \boldsymbol{B})+R e_{m}^{-1} \nabla^{2} \boldsymbol{B} .
\end{aligned}
$$

Here $\boldsymbol{B}$ is the magnetic field, $t$ is time and the magnetic Reynolds number $R e_{m}=d^{2} \omega_{1} / \eta$ where $\eta$ is the magnetic diffusivity. We use the Alfvén scale $B_{0}$ for the magnetic field, where $B_{0}=\sqrt{\rho \mu_{0}} \omega_{1} d$, where $\rho$ is the fluid density and $\mu_{0}$ the magnetic permeability. The Lorentz force is thus formally of the same order as inertial terms in the Navier-Stokes equations

$$
\frac{\partial \boldsymbol{u}}{\partial t}+\boldsymbol{u} \cdot \boldsymbol{\nabla} \boldsymbol{u}=-\nabla p+(\boldsymbol{\nabla} \times \boldsymbol{B}) \times \boldsymbol{B}+R e^{-1} \nabla^{2} \boldsymbol{u}, \quad \boldsymbol{\nabla} \cdot \boldsymbol{u}=0 .
$$

We shall seek steady solutions to equations $(4 \mathrm{a}, \mathrm{b})$, and (5) of the form

$$
\boldsymbol{u}=\left(-r H^{\prime}(z), r G(z), 2 H(z)\right)
$$

and correspondingly

$$
\boldsymbol{B}=\left(-r P^{\prime}(z), r T(z), 2 P(z)\right) .
$$

These clearly satisfy the solenoidal conditions $\boldsymbol{\nabla} \cdot \boldsymbol{u}=0=\boldsymbol{\nabla} \cdot \boldsymbol{B}$. Substitution in (4b) yields respectively for the toroidal and poloidal components

$$
\begin{aligned}
2 H T^{\prime}-2 G^{\prime} P & =R e_{m}^{-1} T^{\prime \prime}, \\
2 H P^{\prime}-2 H^{\prime} P & =R e_{m}^{-1} P^{\prime \prime} .
\end{aligned}
$$

The field is associated with a current density

$$
\boldsymbol{J}=\boldsymbol{\nabla} \times \boldsymbol{B}=\left(-r T^{\prime},-r P^{\prime \prime}, 2 T\right)
$$

and a Lorentz force $\boldsymbol{J} \times \boldsymbol{B}$. Taking the $\phi$-component of equation (5) we have

$$
G^{\prime \prime}=2 \operatorname{Re}\left(H G^{\prime}-G H^{\prime}-P T^{\prime}+P^{\prime} T\right),
$$

while the $\phi$-component of the vorticity equation leads to

$$
H^{\prime \prime \prime \prime}=2 \operatorname{Re}\left(H H^{\prime \prime \prime}+G G^{\prime}-T T^{\prime}-P P^{\prime \prime \prime}\right) .
$$




\section{$2.1 \quad$ Boundary conditions}

The fluid satisfies no slip on the walls so that

$$
H(0)=H(1)=H^{\prime}(0)=H^{\prime}(1)=0, \quad G(0)=1, \quad G(1)=s .
$$

The magnetic field may diffuse out of the fluid layer and so its boundary conditions are less straightforward than those on the velocity. We consider three cases:

2.1.1 Perfectly conducting walls. At an interface, the normal magnetic field and the tangential electric field must be continuous. Inside a perfect conductor the electric field must be zero else it would drive an infinite current. It follows that the tangential electric field on the discs must be zero, which from Ohm's law in the fluid translates to the tangential current must be zero,

$$
\boldsymbol{B} \cdot \boldsymbol{k}=0, \quad \boldsymbol{J} \times \boldsymbol{k}=\mathbf{0},
$$

where $\boldsymbol{k}$ is a unit vector in the $z$-direction. From the form of equations (7) and (10) this requires

$$
P=0 \quad \text { and } \quad T^{\prime}=0 \quad \text { on } z=0,1 .
$$

We note that the other tangential current component $P^{\prime \prime}$ is automatically zero on the walls due to the nonslip condition and equation (8).

2.1.2 Insulating boundaries. If the exterior is an insulator, then clearly $\boldsymbol{J}=0$ within it, which requires from (10) $T \equiv 0$ and $P^{\prime \prime} \equiv 0$ in $z<0$ and $z>1$. At the boundaries the magnetic field is continuous and so the appropriate conditions are

$$
T=T^{\prime}=0 \quad \text { on } z=0,1 .
$$

Inside the insulator we can consistently have $P^{\prime \prime}=0$ or $P=A+C z$. With regard to (7) this corresponds to a uniform field in the $z$ direction of strength $A$ and a magnetic neutral point of strength $C$.

2.1.3 Ferromagnetic boundaries. The VKS experiment was successful when the rotors were made of iron with a high magnetic permeability $\mu \simeq 100 \mu_{0}$, (Monchaux et al. 2009). At a boundary where the permeability is discontinuous, the normal component of $\boldsymbol{B}$ and the tangential components of $\boldsymbol{B} / \mu$ must be continuous. As the tangential $\boldsymbol{B}$ within the iron cannot be excessive, this requires that within the liquid, the tangential component of $\boldsymbol{B}$ should be small. From (7), as $\mu_{r} \equiv \mu / \mu_{0} \rightarrow \infty$, this translates to

$$
P^{\prime}=0 \quad \text { and } \quad T=0 \quad \text { on } z=0,1 .
$$

\section{$2.2 \quad$ Numerical methods}

We solve the ODE system (8), (11) and (12) together with (13) and one of (15), (16) or (17) using the MATLAB ODE solver bvp4c, described in Shampine et al. (2003). For the eigenvalue problem, when it reduces to Sturm-Liouville form we employ the package of Ledoux et al. (2005). Otherwise, we introduce an extra equation $\lambda^{\prime}=0$ and an arbitrary inhomogeneous, possibly complex, boundary condition normalising the eigenfunction. We use 2000 grid points between $z=0,1$ and work to a tolerance of at most $10^{-6}$ in all cases. 

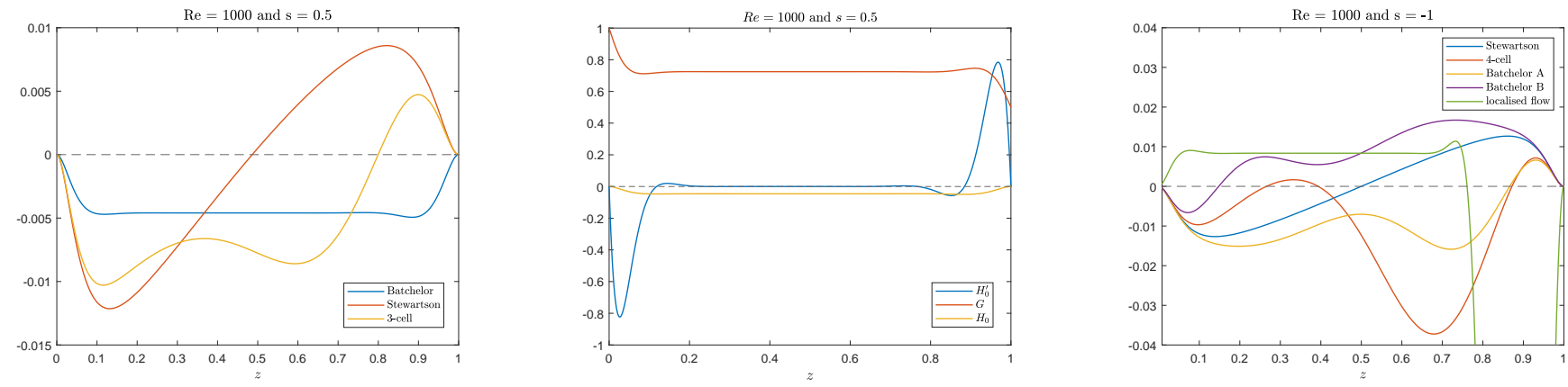

Figure 2. Multiple solution branches for $B \equiv 0$ at $R e=1000$ and $s=0.5$ (left) and $s=-1$ (right), $H(z)$ is drawn for each solution. Middle: Flow on the Batchelor branch (blue in left panel) for $R_{e}=1000, s=0.5$, with $H_{0}=10 H$. Away from the walls, the flow is close to solid body rotation, with the $z$-velocity directed towards each wall. The blue curve in the left panel corresponds to the yellow one in the middle.

\subsection{Hydrodynamic flows}

Physically, in the absence of magnetic field, variations in the swirl velocity $G$ lead to radial pressure gradients which drive a poloidal flow. $H$ may be of single sign or may reverse somewhere in the interior.

In this paper we shall regard $s$ and $R e$ as given and investigate the resultant solutions for differing values of $R e_{m}$. Nevertheless, we must take into account the existence of different hydrodynamic solution branches. For moderately high Re the Batchelor (1951) branch, shown in the middle panel of figure 2, is characterised by a core flow close to solid body rotation with Ekman-like layers on the walls. Here $H$ is of one sign and $H^{\prime}$ has opposite signs on the walls, so that flow is directed away from one wall and towards the other. In contrast, on the Stewartson (1953) branch, for the same parameters $G \simeq 0$ away from the walls and $H^{\prime}<0$ on each wall. The $z$-velocity is therefore directed towards each wall. These two flows are found to exhibit different dynamo behaviour. The Stewartson branch is found to give rise to a higher kinematic growth rate. However, in $\S 5$ we argue that it is unlikely to function as a dynamo when the discs are finite. There is a third solution, which behaves like the Stewartson branch at the walls. When $s<0$ even more branches can occur. For $R e=1000$ ssand $s=-1$, five different solutions are shown in the right panel of figure 2. Only the Stewartson solution retains the anti-symmetry of the configuration, and the others may be reflected to provide four other solutions. All these solutions have $H^{\prime}<0$ on both walls, which we will see below improves dynamo action.

\section{Kinematic Dynamos}

When the field is very weak, the Lorentz force can be neglected in (5) and we have a pure Von Kármán flow. In this section $G$ and $H$ are regarded as given. We seek growing solutions for the magnetic field of the form $\boldsymbol{B}=\left(r P^{\prime}, r T,-2 r P\right) \exp (\lambda t)$ where the growth rate $\lambda$ may be complex. We have the eigenvalue problem

$$
\begin{aligned}
\lambda T+2 H T^{\prime}-2 G^{\prime} P & =R e_{m}^{-1} T^{\prime \prime}, \\
\lambda P+2 H P^{\prime}-2 H^{\prime} P & =R e_{m}^{-1} P^{\prime \prime}
\end{aligned}
$$

with the boundary conditions of (15), (16) or (17). We note that (18) and (19) are only weakly coupled as there is no $T$ in the second equation. This reflects the common difficulty in dynamo theory of generating poloidal from toroidal field. In contrast, differential rotation $\left(G^{\prime}\right)$ can wind poloidal field $(P)$ into toroidal field $(T)$. 

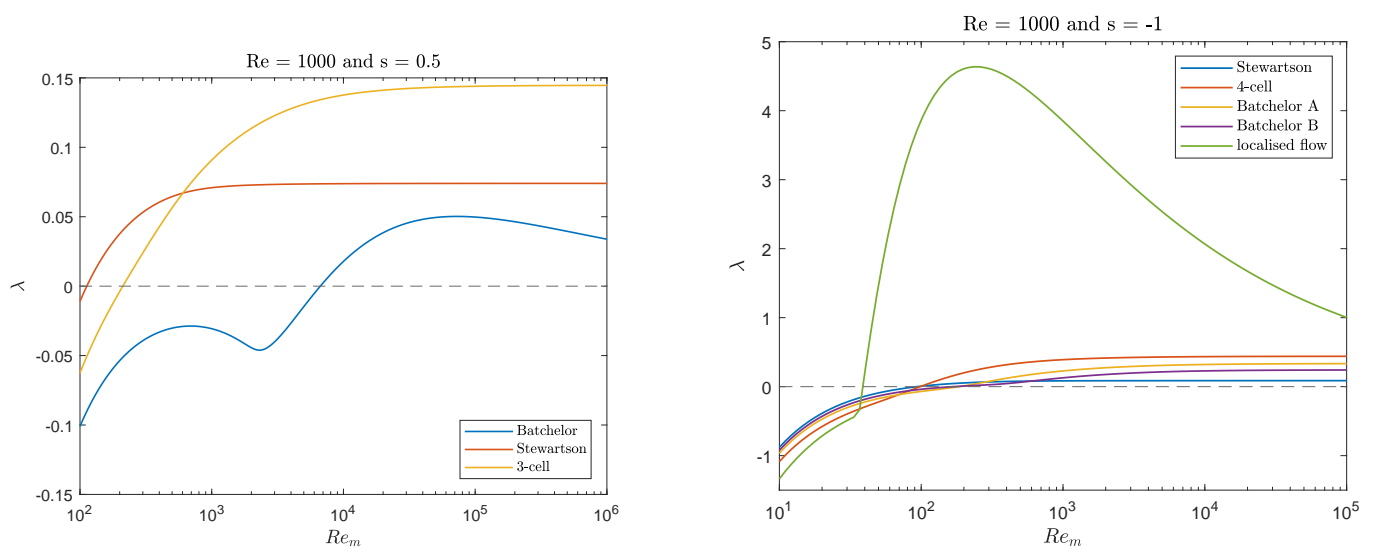

Figure 3. Growth rates for perfectly conducting boundaries, for different branches at $R e=1000$. Left: $s=0.5$, Right: $s=-1$. The Batchelor branch (blue) in the left is slow, while the Stewartson (red) and 3-cell (yellow) branches are fast, in agreement with (23). All the branches on the right are fast. However, in $\S 5$ we find only the slow blue branch has an outward energy flux.

\subsection{Perfectly conducting dynamo}

If the boundary conditions are $P=T^{\prime}=0$ on the boundaries $z=0,1$, the $T$-equation decouples and we have a single ODE to solve,

$$
\lambda P+2 H P^{\prime}-2 H^{\prime} P=R e_{m}^{-1} P^{\prime \prime}, \quad P(0)=P(1)=0 .
$$

This is an unusually simple dynamo problem, which can be written in Sturm-Liouville form. It follows that all the eigenvalues $\lambda\left(R e_{m}\right)$ are real. Only the poloidal velocity is important, although it should not be forgotten that $H \neq 0$ only because of the existence of $G$ in (5). Once $\lambda$ and $P(z)$ have been found, $T(z)$ can be found from the inhomogeneous problem with forcing $P G^{\prime}$

$$
\lambda T+2 H T^{\prime}-2 G^{\prime} P=R_{m}^{-1} T^{\prime \prime}, \quad T^{\prime}(0)=T^{\prime}(1)=0 .
$$

It is worth noting that there is another family of eigenvalues, for which $P \equiv 0$ and $\lambda$ and $T$ are determined from (21). These modes can be shown always to have $\lambda \leqslant 0$ and so are not dynamos. The trivial solution with $\lambda=0$ and $T$ constant should be recognised. This corresponds to a uniform current in the $z$-direction in the fluid, which then spreads out as a radial surface current along the perfectly conducting walls. It is not a growing mode, but it will be important when we consider nonlinear steady-states in section 4 . It also demonstrates that sometimes our similarity solutions can be driven by conditions as $r \rightarrow \infty$.

The eigenvalues for (20) are easily found numerically. For the flows of figure 2 corresponding to $R e=1000$ and $s=0.5$ or $s=-1$, the largest eigenvalue is given in figure 3. A growing mode is found for $R e_{m}>109.5$ for the Stewartson branch when $s=0.5$. When $s=-1$, with the increased shear this value is reduced, and the hybrid branch is the first to manifest growth. As $R e_{m} \rightarrow \infty$, it is found that $\lambda$ may either tend to a constant or, for the Batchelor branch, $\lambda \rightarrow 0$ as $R e_{m}^{-1 / 3}$. We confirm this latter behaviour analytically.

As $R e_{m} \rightarrow \infty$, away from the boundaries we see that $P \propto H$ is a solution to (20) for small $\lambda$. However, near say $z=0$, we have $H \sim \alpha z^{2}$, where $\alpha$ is determined from the Navier-Stokes solution (5). The righthand side of $(20)$ is therefore non-zero, and so the solution for $P$ must adapt across a layer of thickness $\delta$ say. In this layer, if we introduce a scaled coordinate $Z=z / \delta,(20)$ becomes

$$
\begin{gathered}
\lambda P+2 \alpha \delta Z^{2} P^{\prime}-4 \alpha \delta Z P=\frac{P^{\prime \prime}}{R e_{m} \delta^{2}}, \\
P(0)=0, \quad P \sim C Z^{2} \quad \text { as } Z \rightarrow \infty,
\end{gathered}
$$


where ' now denotes $\mathrm{d} / \mathrm{d} Z$. This suggests the balance $\delta=R e_{m}^{-1 / 3}$, so that

$$
\begin{gathered}
\mu P+2 \alpha Z^{2} P^{\prime}-4 \alpha Z P=P^{\prime \prime}, \\
P(0)=0, \quad P \sim C Z^{2} \quad \text { as } \quad Z \rightarrow \infty,
\end{gathered}
$$

where $\mu=\lambda R e_{m}^{1 / 3}$. An essentially identical equation is appropriate next to the upper disc at $z=1$, where $H \sim \beta(1-z)^{2}$, in terms of the scaled variable $Z=\operatorname{Re}_{m}^{1 / 3}(1-z)$. Equation (22) can be solved in terms of triconfluent Heun functions. The crucial result is that as $Z \rightarrow \infty$, the two independent solutions scale as $P^{\prime} \sim Z$ and $P^{\prime} \sim \exp \left(\frac{2}{3} \alpha Z^{3}\right)$. Thus if $\alpha<0$ there is no trouble matching with the field away from the boundary, for any value of $\mu$. However, if $\alpha>0$, then a solution is only possible if the exponentially growing solution has zero coefficient, which will only happen for discrete values of $\mu$. Similarly near $z=1$, the layer is unconstrained if $\beta>0$ but determines the eigenvalue if $\beta<0$. Now for the Batchelor flow in figure 2, near $z=0$ we find $\alpha<0$, whereas on $z=1$ we have $\beta<0$. In this case the growth rate $\mu$ is determined by the layer on the upper plate, while the layer on the lower plate is passive. If $s>1$, these roles are reversed. In general we conclude that for flows of this type it is the layer on the more slowly rotating disc which determines the growth rate.

The above analysis relies on the poloidal flow $H$ being towards one of the plates and away from the other, as occurs in the Batchelor branch of figure 2. For the Stewartson branch in the left and all branches on the right of figure 2 , the $H$-flow is towards each boundary, and so the wall layers do not constrain $\lambda$. However, there is now necessarily an internal value $z=z_{0}$ at which $H$ vanishes. Looking at equation (20), we can see that as $R e_{m} \rightarrow \infty$, a regular solution would require $\lambda=2 H^{\prime}\left(z_{0}\right)$. Such a dynamo would be fast as $\lambda \nrightarrow t 0$ as $R e_{m} \rightarrow \infty$. In this limit we can integrate (20) to find

$$
P=H(z) \exp \left[-H^{\prime}\left(z_{0}\right) \int^{z} \frac{\mathrm{d} Z}{H(Z)}\right] \text {. }
$$

It can be confirmed that $P(z)$ is regular provided $H(z)$ is negative for $0<z<z_{0}$ and positive for $z_{0}<z<1$ with $\alpha<0, \beta>0$ and $\lambda=2 H^{\prime}\left(z_{0}\right)>0$. Cases with fast dynamos are shown in figure 3 .

For given flow parameters $R e$ and $s$ there is a critical magnetic Reynolds number, $R e_{m}=R_{c}$, above which dynamo action occurs. The behaviour of $R_{c}$ as $R e$ and $s$ vary is shown in figure 4 for perfectly conducting walls. When $s=1$ the flow is a solid body rotation which does not drive a dynamo, and so $R_{c} \rightarrow \infty$ as $s \rightarrow 1$ for all Re. A kink is observable on one curve. This relates to a switching of the underlying flow branch. It can be seen that the lowest $R_{c}$ occur for counterrotating discs, when $s<0$. The increased shear improves dynamo action. It should be remembered that when $|s|>1$, the problem can be rescaled with respect to the greater angular velocity to a mathematically equivalent problem with $|s|<1$ with corresponding reductions in $R e$ and $R e_{m}$. It it also the case that increasing $R e$ reduces $R_{c}$ until an asymptotic flow structure is reached. For this reason, we shall concentrate below on moderately high $R e$.

\subsection{Dynamos with insulating walls}

When the walls are insulating, equation (15) applies and we have two boundary conditions on $T$ and none on $P$. We could eliminate $P$ between (18) and (19) to obtain a fourth order ODE for $T$, but it is as easy to deal with the two second order ODEs. A parallel could be drawn with writing the 2D vorticity equation in terms of a streamfunction, when there are two boundary conditions on the streamfunction but none on the vorticity. This case behaves in a manner more common for kinematic dynamos: the eigenvalues need not be real, while both toroidal and poloidal field components are important.

The growth rate for the Stewartson branch for $R e=1000$ and $s=-1$ is shown in figure 5(a), with a critical $R e_{m} \simeq 108$. It will be shown below that subcritical nonlinear solutions exist considerably below this value, even for $R e_{m} \gtrsim 25$.

The eigenfunctions for perfectly conducting, insulating and ferromagnetic walls are shown in figure 6 for $s=-1$ and $R e=100$ for $R e_{m}=100$. In each case the unstable mode is symmetric. It should be 

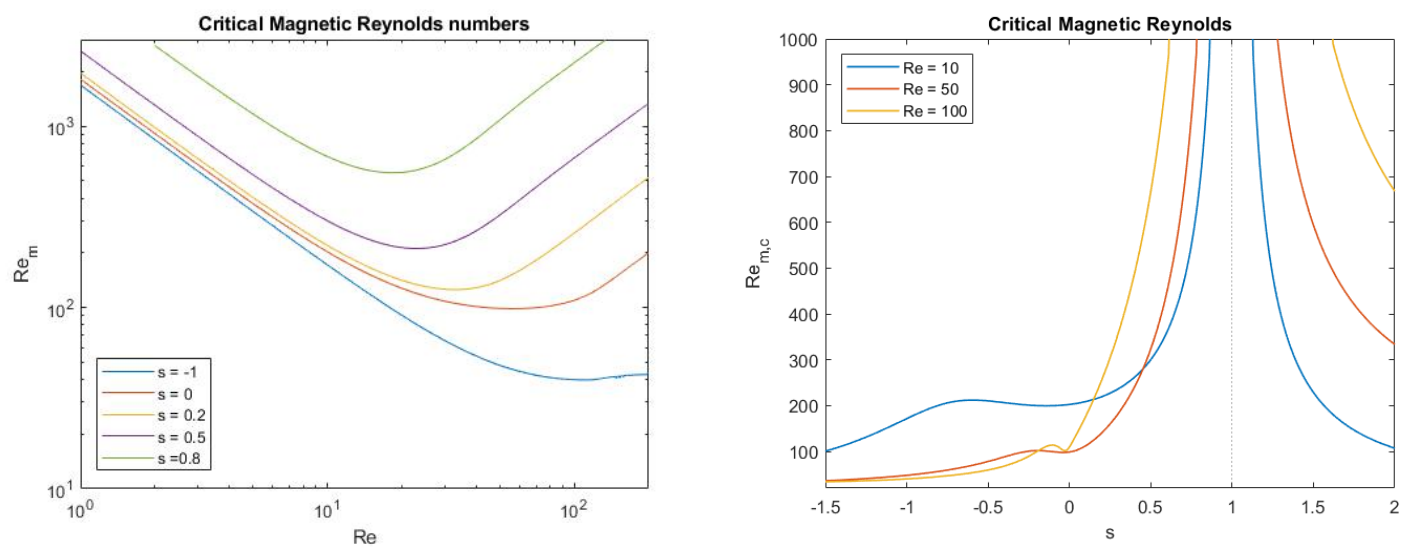

Figure 4. Critical magnetic Reynolds number as a function of Re and $s$ for perfectly conducting boundaries. Left: Re varies for several $s$. Right: $s$ varies for several $R e$.
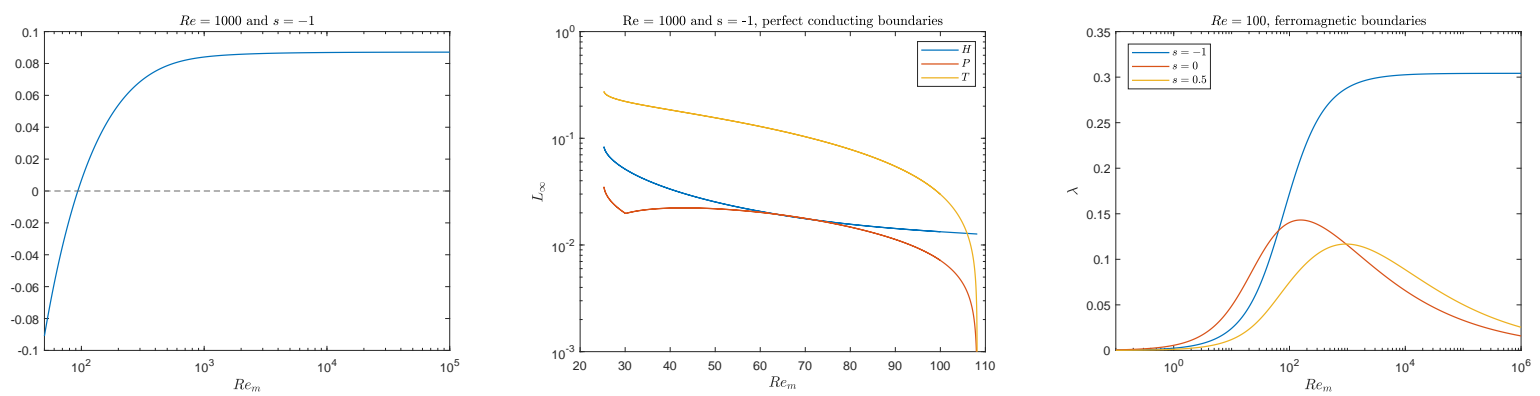

Figure 5. Left: Growth rate for Stewartson branch, $R e=1000$ and $s=-1$. Middle: Subcritical saturated states for $R e=1000, s=-1$. As $R e_{m}$ approaches critical value of the left panel, the field is quenched. Right: $R e=100$ and perfectly ferromagnetic boundaries for $s=-1,0,0.5$. Growth occurs for all $R e_{m}$. The dynamo is fast for $s=-1$, but $s=0$ has the largest growth rate as $R e_{m} \rightarrow 0$.
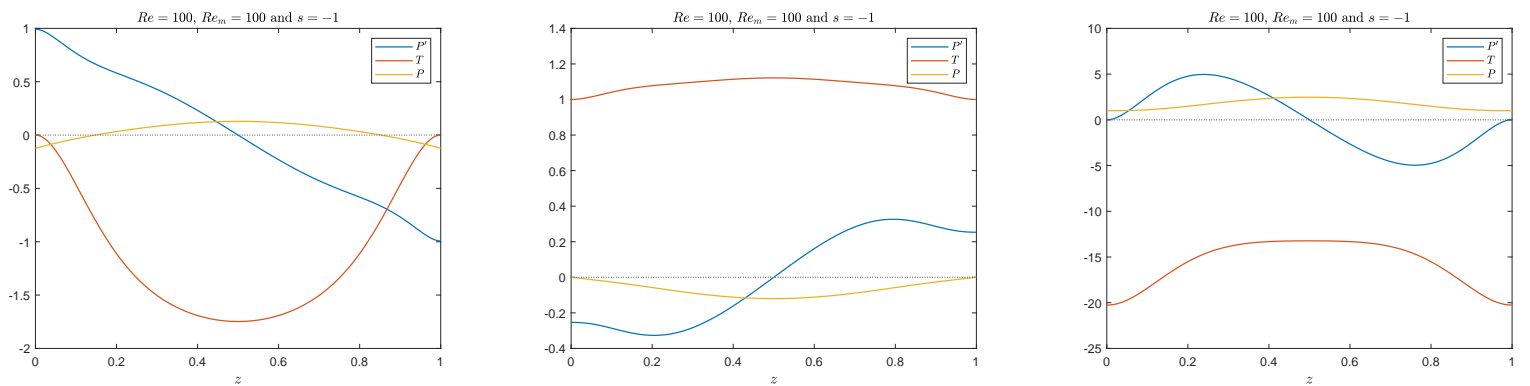

Figure 6. The growing eigenfunctions for $R e=100=R e_{m}$ and $s=-1$. Left: insulating, middle: perfectly conducting walls, right: ferromagnetic boundary conditions

remembered that the scale and sign of the field are arbitrary at this stage.

\subsection{Ferromagnetic dynamo}

The VKS experiment found a dynamo when the rotors were made of iron with a relative permeability $\mu_{r} \simeq 100$. We might therefore anticipate that the boundary condition (17) would favour dynamo action. Indeed, we find numerically that any $R e_{m}>0$ drives a dynamo. This can be shown by writing the SturmLiouville system in variational form, or more directly if we assume $P>0$ for $0 \leqslant z \leqslant 1$. Dividing equation (8) by $P^{2}$ and integrating across the domain, it follows that

$$
\lambda \int_{0}^{1} \frac{\mathrm{d} z}{P}=2\left[\frac{H}{P}\right]_{0}^{1}+R e_{m}^{-1} \int_{0}^{1} \frac{P^{\prime \prime}}{P^{2}} \mathrm{~d} z=2 R e_{m}^{-1} \int_{0}^{1} \frac{\left(P^{\prime}\right)^{2}}{P^{3}} \mathrm{~d} z
$$



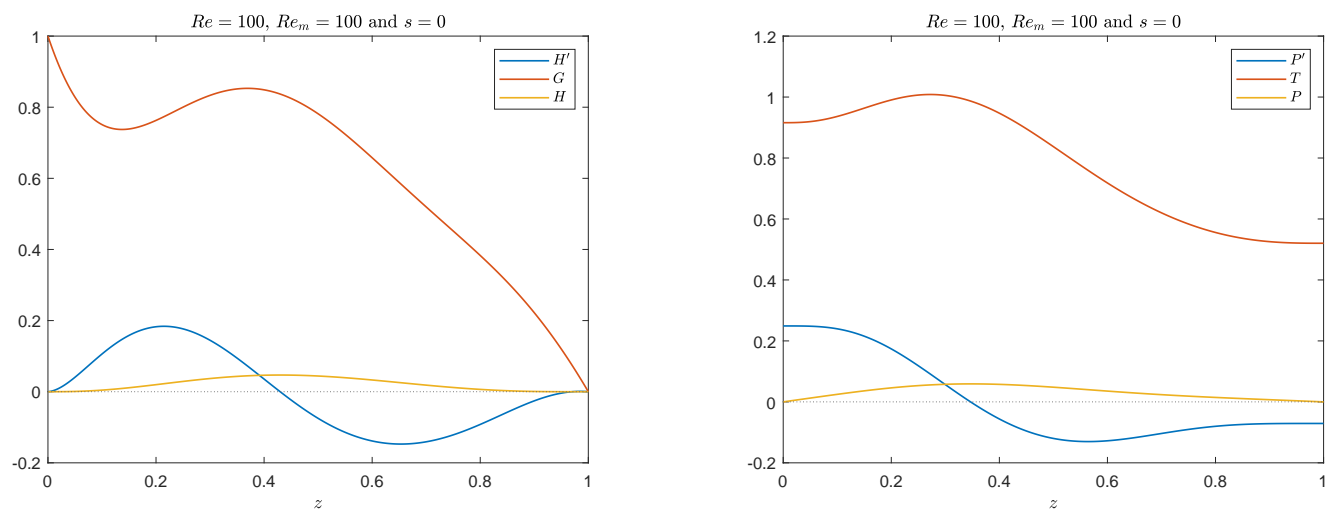

Figure 7. Saturated states for perfectly conducting plates at $R e=100, R e_{m}=100$ and $s=0$. Left: flow, Right: magnetic field.

where we integrate by parts and employ the boundary conditions, $H=0$ and $P^{\prime}=0$. As $P>0$ by assumption, it follows that $\lambda>0$ and we have growth. As $R e_{m} \rightarrow 0$, we can readily obtain the solution as a regular perturbation series

$$
P=1-2 R e_{m} \int_{0}^{z} H(s) \mathrm{d} s+\mathrm{O}\left(\operatorname{Re}_{m}^{2}\right), \quad \lambda=8 R e_{m} \int_{0}^{1} H^{2} \mathrm{~d} z+\mathrm{O}\left(R e_{m}^{2}\right) .
$$

This growth rate is confirmed numerically. The variation of $\lambda$ with $R e_{m}$ is shown in figure $5(\mathrm{c})$ for 3 values of $s$. It is surprising to find a dynamo for small $R e_{m}$, but the physical mechanism is clear. Constant $P$ corresponds to a uniform field in the $z$-direction, and this is distorted by the poloidal flow $H$ to give a weak radial field, and then by the toroidal flow $G$ to give a weak azimuthal field. The ferromagnetic boundaries then amplify these fields within the iron boundary, generating a large $z$-component, which feeds back to the fluid by continuity. Thus the dynamo at very low $R e_{m}$ is a consequence of the infinite permeability of the boundary. If the relative permeability of the boundary, $\mu_{r}$ is large but finite, the critical $R e_{m}$ scales as $\mu_{r}^{-1 / 2}$.

In broad terms, these results are consistent with the experimental observation that constructing the rotating boundaries from ferromagnetic material improves the dynamo action.

\section{Nonlinear saturated dynamos}

We have demonstrated that the von-Kármán velocity fields support kinematic dynamos in an unbounded domain. What happens once the field grows is not clear, a priori. Periodic oscillations or more complicated time-dependent behaviour could occur, even for our simple laminar flows. It is also possible however, that steady hydromagnetic equilibria may occur with the structure given by (3) and (7). It is a great advantage of this ansatz that we can easily investigate the fully nonlinear behaviour, without further approximation. In this section we therefore seek solutions to the system (11), (12) and (8). Various solution branches are found, depending to some extent on which initial flow branch is used.

In figure 7, a saturated equilibrium is drawn for $R e=100, R e_{m}=100$ and $s=0$. As might be expected, there is more activity near the rotating disc than the stationary one. Once an equilibrium has been found, its solution branch may be tracked by slowly varying the parameters. For the case of equal and opposite rotation rates $(s=-1)$, the behaviour of the maximum values of $P, T$ and $H$ is shown in figure 8 for $R e=100$ as $R e_{m}$ varies. $G$ always attains its maximum on the boundary and so is not drawn. In the left panel, the boundaries are perfectly conducting. As $R e_{m}$ increases, at a certain value the solution branch terminates at an essentially hydrodynamic flow with $P \equiv 0$ and $T$ uniform. These solutions have a uniform $z$-current in the fluid which then spreads out as a radial surface current on the boundaries. It is not obvious why this branch terminates at that particular value of constant $T$. Such solutions feel somewhat artificial and they do not exist for the insulating case. Equilibria are found for $R e_{m} \geqslant 70$, even though the critical 

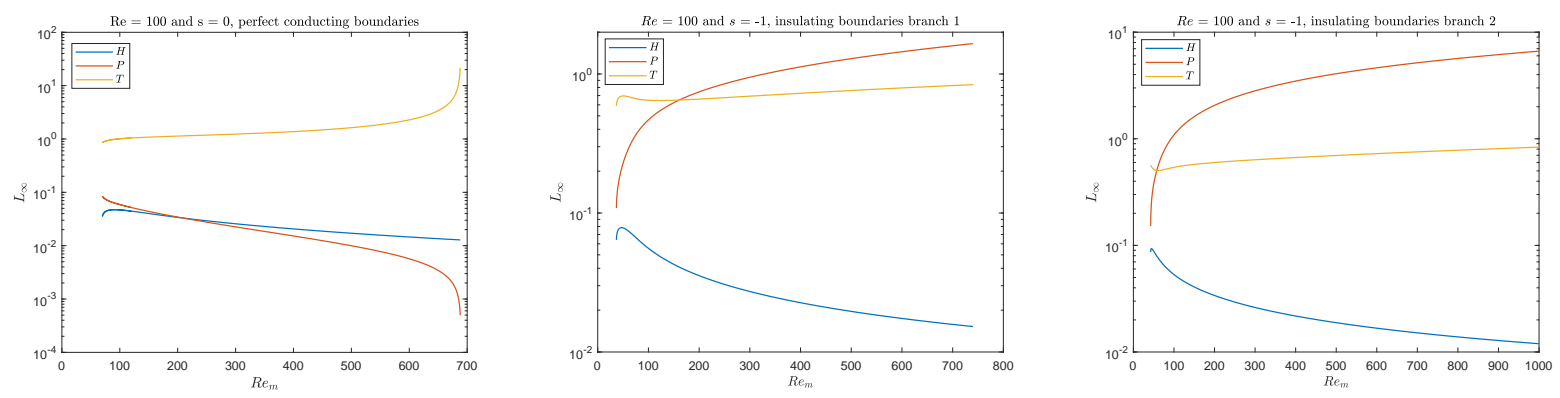

Figure 8. $L_{\infty}$-norm of saturated solution as a function of $R e_{m}$ for $R e=100, s=-1$. Left: Perfectly conducting boundaries. As $R e_{m}$ increases the branch terminates with $P \rightarrow 0$, constant $T \neq 0$. Non-zero fields exist for $R e_{m}=70<R_{c} \simeq 109.5$, suggesting that the dynamo bifurcation is subcritical. Middle: \& Right: insulating boundaries. Again subcritical solutions are found for $R e_{m} \geqslant 37$.
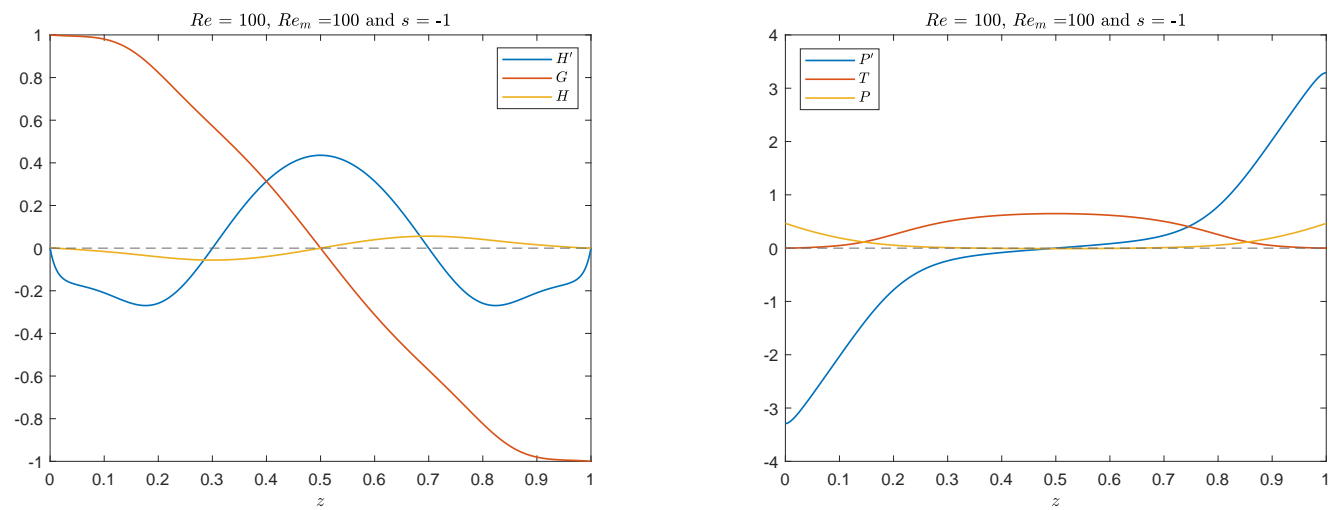

Figure 9. Symmetric steady state for $R e=100, s=-1$ and $R e_{m}=140$ and insulating boundaries. Left: flow, right: magnetic field. The field is even about $z=0$, while the flow is odd.

value for a kinematic dynamo is $R e_{m} \simeq 109.5$. The dynamo instability is therefore subcritical and the system exhibits hysteresis.

The subcritical behaviour is illustrated in figure 5(b) for insulating boundaries with $R e=1000$ and $s=-1$. Equilibria are found for $R e_{m} \gtrsim 30$ with an amplitude which tends to zero as the critical $R e_{m}$ is approached from below. The kink in the curve is an artefact of the maximum norm. If $P$ has two maxima then the position of the overall maximum may flip as $R e_{m}$ varies.

In the right two panels of figure 8 , two solution branches are shown for the insulating case. One of these displays the expected symmetry about $z=0.5$, but in the other, symmetry is broken. These branches continue for large $R e_{m}$. Once more, hydromagnetic solutions are found as $R e_{m}$ decreases below $R_{c}$, the critical value for a kinematic dynamo.

In the insulating case, the solutions for $R e_{m}=100$ are drawn in figure 9. Already the signs of an asymptotic structure are there. There is an active region about $z=0$ for the symmetric case and near the boundary for the nonsymmetric case, of thickness $\mathrm{O}\left(R e_{m}^{-1 / 2}\right)$. Outside this region, $G$ attains its constant wall value, while $P$ is quadratic in $z$. This solution does not satisfy $P^{\prime \prime}=0$ on the actual boundaries, and so there is an additional thin layer near these boundaries, which is most visible in the blue curves. As $R e_{m}$ increases further, the structure becomes more pronounced.

\section{$5 \quad$ Are these real dynamos?}

We have demonstrated that fields of the form (7) can be supported by flows of the form (3) in a selfsustaining manner even in the nonlinear regime. We have referred to this as dynamo action. However, our domain is unbounded in the radial direction and both the flow and magnetic field grow linearly with $r$. In this section we discuss the extent to which these solutions can be considered dynamos in the usual sense.

First of all, from a mathematical perspective, the kinematic dynamo problem can be rewritten in a 

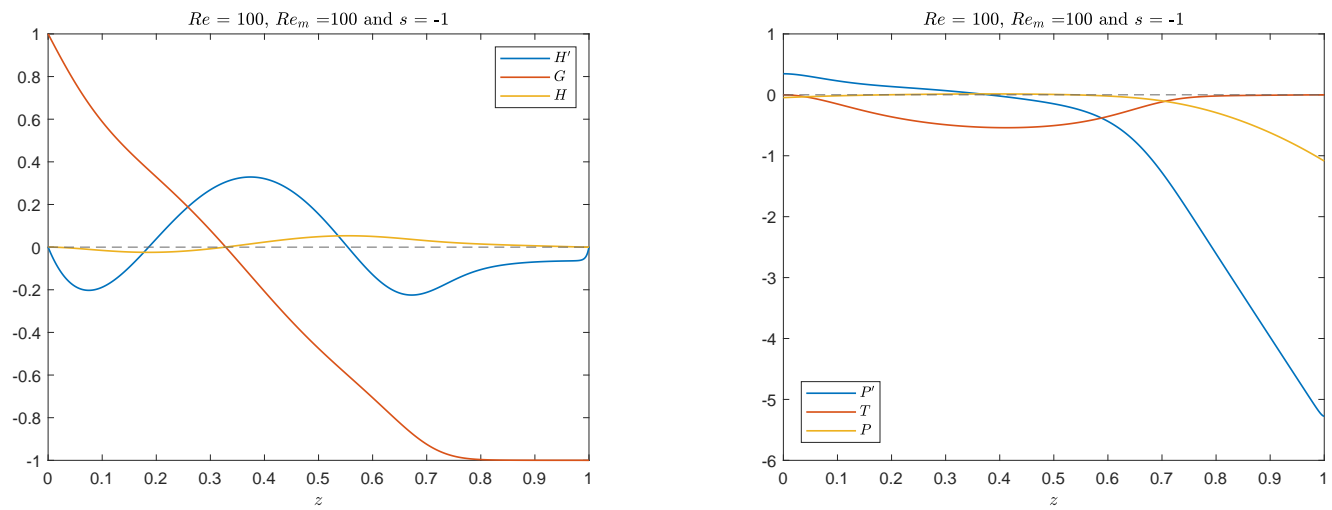

Figure 10. Second, symmetry-breaking steady state for $R e=100, s=-1$ and $R e_{m}=140$ and insulating boundaries.

bounded domain, $0<r<R$, together with some homogeneous boundary conditions on $r=R$, say

$$
\frac{\partial B_{z}}{\partial r}=0, \quad \frac{\partial}{\partial r}\left(\frac{B_{\theta}}{r}\right)=0
$$

These boundary conditions do not necessarily drive a solution which grows with $r$; however neither do they preclude it. Our solution will then hold in $0<r<R$, with these boundary conditions. We note that as $B_{r} \neq 0$ on $r=R$, there will also be (potential) field in $r>R$. This field will be bounded and so the dynamo solution makes mathematical sense, given these boundary conditions.

However, the above boundary conditions would be difficult to arrange in practice, and so we should consider the relevance to a physical problem. In particular, if we were to replace our infinite discs with large but finite ones, and embed the entire system in a stationary box with decaying field outside it, would it still exhibit growing solutions?

This is difficult to answer definitively, as our simplifying structure would naturally be destroyed by these distant boundaries. However, what we can easily examine is whether our solutions involve an inward or outward flow of energy at large $r$. If our solutions involve an influx of magnetic energy from infinity, then while they describe interesting local hydromagnetic equilibria, we could not regard them as local generators of magnetic field. Conversely, if our solutions involve an outward energy flux, then field is being generated at low $r$ and transported towards infinity. It is then arguable that our solutions could approximate the local behaviour of a larger dynamo structure. We shall therefore assume that our solution is valid to leading order for $r<R$ and investigate the fluxes across the boundary $r=R$. Note that $R$ may be smaller than the actual disc radius. It represents a value below which our solution is assumed to be a good approximation to the full solution. We shall also assume $R \gg 1$, so that the discs are larger than their separation distance.

If we begin with the purely hydrodynamic problem, it is intuitively obvious that the flow is being driven by the differential rotation of the discs and opposed by the distant boundaries. We would therefore expect the von Kármán flows to apply for small $r$. Explicitly, if we take the scalar product of the Navier-Stokes equation with $\boldsymbol{u}$ and integrate over $0<z<1,0<r<R$, we obtain

$$
\frac{\mathrm{d}}{\mathrm{d} t} \int \frac{1}{2}|\boldsymbol{u}|^{2} \mathrm{~d} V=-\oint\left(p+\frac{1}{2}|\boldsymbol{u}|^{2}\right) \boldsymbol{u} \cdot \boldsymbol{n} \mathrm{d} S-\text { viscous terms. }
$$

The viscous terms include both the driving stress on the discs and the volume dissipation. Now on the cylindrical boundary at $r=R$ we have $\boldsymbol{u} \cdot \boldsymbol{n}=-R H^{\prime}(z)$, while $|\boldsymbol{u}|^{2}=R^{2} H^{\prime 2}+R^{2} G^{2}+H^{2} \simeq R^{2}\left(G^{2}+H^{\prime 2}\right)$ for large $R$.

Substituting (3) into (5), we find from the $z$-component that $p_{z}$ is $\mathrm{O}(1)$ as $r \rightarrow \infty$, while from the $r$-component we have $p_{r}=\mathrm{O}(r)$. It follows that the pressure in $(27)$ is a constant times $R^{2}$ plus $\mathrm{O}(1)$ terms. The pressure therefore does not contribute at leading order as the integral of $H^{\prime}$ vanishes since 
$H=0$ on both discs. Thus the kinetic energy flux across $r=R$ is determined to leading order by

$$
\widehat{K} \equiv \pi R^{4} K \quad \text { where } \quad K=\int_{0}^{1}\left(G^{2}+H^{\prime 2}\right) H^{\prime} \mathrm{d} z .
$$

If $K<0$ then energy is flowing outwards. This we find to be the case for all parameter values. Thus the von Kármán flow can be expected to be a good model of the flow between two discs inside a larger container.

We now consider the magnetic energy flux. From Faraday's Law, $\nabla \times \boldsymbol{E}=-\boldsymbol{B}_{t}$, taking the scalar product with $\boldsymbol{B}$ and integrating over our domain, we have

$$
\frac{\mathrm{d}}{\mathrm{d} t} \int \frac{1}{2}|\boldsymbol{B}|^{2} \mathrm{~d} V=\oint(\boldsymbol{B} \times \boldsymbol{E}) \cdot \boldsymbol{n} \mathrm{d} S-\int(\boldsymbol{\nabla} \times \boldsymbol{B}) \cdot \boldsymbol{E} \mathrm{d} V,
$$

where $\boldsymbol{E}$ denotes the electric field. Using the non-dimensional Ohm's Law, $\boldsymbol{E}=\boldsymbol{J}-R_{m} \boldsymbol{u} \times \boldsymbol{B}$ and Ampere's Law, $\boldsymbol{\nabla} \times \boldsymbol{B}=\boldsymbol{J}$, the last term contributes the Joule dissipation rate $-|\boldsymbol{J}|^{2}$ and the work done by the fluid $-\boldsymbol{u} \cdot(\boldsymbol{J} \times \boldsymbol{B})$. The boundary integral of the Poynting vector $\boldsymbol{E} \times \boldsymbol{B}$ indicates the direction of the energy flux. Now for large $r, \boldsymbol{J} \propto r$, while $\boldsymbol{u} \times \boldsymbol{B} \propto r^{2}$. Furthermore, from (10) and (7), $\boldsymbol{J} \times \boldsymbol{B}$ is to leading order perpendicular to the radial direction. The dominant term at large $r$ is thus

$$
\widehat{M}=\oint(\boldsymbol{B} \times \boldsymbol{E}) \cdot \boldsymbol{n} \mathrm{d} S \simeq-R_{m} \oint\left[|\boldsymbol{B}|^{2} \boldsymbol{u}-(\boldsymbol{u} \cdot \boldsymbol{B}) \boldsymbol{B}\right] \cdot \boldsymbol{n} \mathrm{d} S .
$$

If $\widehat{M}>0$ then energy is flowing in across the boundary at large $r$, but if $\widehat{M}<0$, the energy flow is outwards and we consider the dynamo functional. At large $r=R$, recalling $\boldsymbol{u}=\left(-r H^{\prime}, r G, 2 H\right)$ and $\boldsymbol{B}=\left(-r P^{\prime}, r T, 2 P\right)$, we have $|\boldsymbol{B}|^{2}=R^{2}\left(P^{\prime 2}+T^{2}\right)+\mathrm{O}(1)$, the magnetic energy flux $\widehat{M}=2 \pi R^{4} R_{m} M$ where

$$
M=\int_{0}^{1}\left[\left(P^{\prime 2}+T^{2}\right) H^{\prime}+\left(-P^{\prime}\right)\left(P^{\prime} H^{\prime}+T G\right)\right] \mathrm{d} z=\int_{0}^{1}\left[T^{2} H^{\prime}-P^{\prime} T G\right] \mathrm{d} z .
$$

Note that the azimuthal components of $\boldsymbol{u}$ and $\boldsymbol{B}$ contribute to $M$.

For each of our solutions, we can evaluate $M$. We find that some of our solutions have $M>0$, and are not real dynamos by this criterion. The fast dynamos are in this category, as illustrated in the left of figure (11). The kinematic growth rate for the Stewartson branch with $s=-1$ at $R e=500$ is depicted above the corresponding inwards magnetic energy flux. We can also see that this flux increases at large $R_{m}$ because of the boundary layer structure. However, more interestingly, for some flows we find $M<0$ indicating outwards energy flux, and these we consider to be local dynamos. The case on the right of figure (11) relates to the Batchelor branch for $s=0.5$ at $R e=500$ and is of particular interest, as the sign of $M$ varies with $R e_{m}$. Usually, $M$ remains single-signed as $R e_{m}$ varies, but here three different regimes can be identified.

The nonlinear equilibria can also be categorised in this way. In the left panel of figure $12 \mathrm{~K}$ and $M$ are shown as functions of $R e_{m}$ for the saturated solutions between insulating discs at $R e=100$ and $s=-1$. The kinetic energy flux is outwards, but above the dynamo threshold we find that there is an inwards magnetic flux from infinity. The right-hand panel is for $R e=1000$ and $s=0.8$ and insulating discs. In this case, we have an outwards energy flux at all $R e_{m}$. It appears that breaking the $z$-symmetry of the flow is more likely to lead to an outwards magnetic energy flux. This is probably because when $s \neq-1$ there is non-zero swirl away from the discs at least in the Batchelor branch, which contributes to the maintenance of the dynamo. This observation may be important for the design of experiments.

When our discs are embedded in a larger container, magnetic energy is generated between the discs will be transported outwards where it will be dissipated in the less favourable environment. It should not be forgotten that $M<0$ does not guarantee a global dynamo. Indeed, we know that if the remainder of the configuration is exactly axisymmetric than Cowling's theorem will apply. That is a topological result, and 

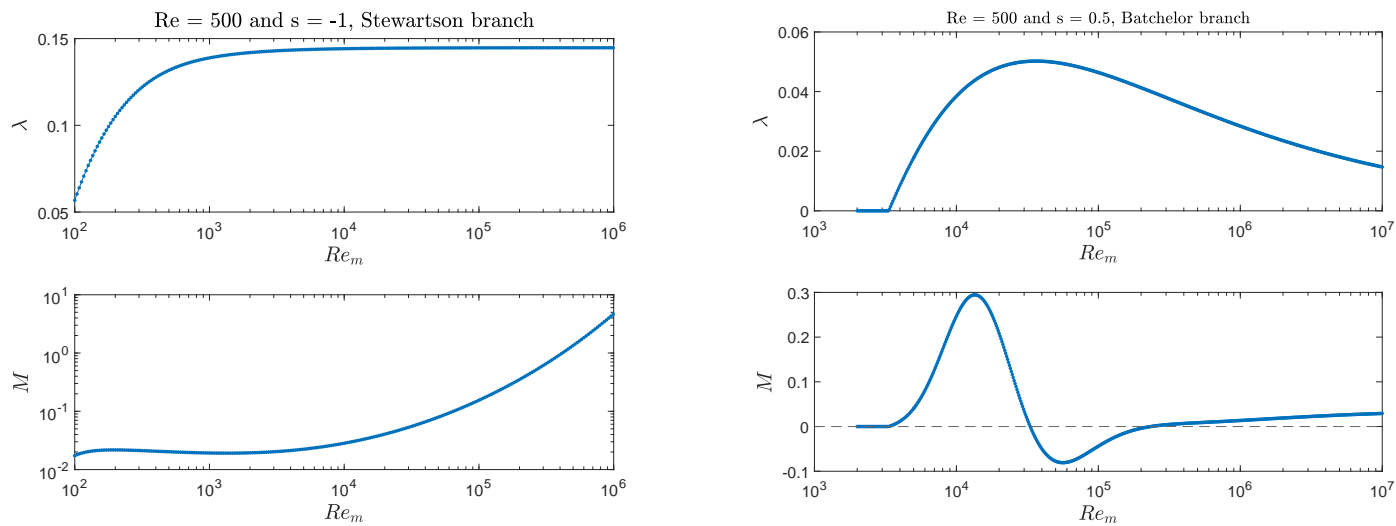

Figure 11. Magnetic energy flux, $M$, for large $r$ and perfectly conducting discs. Left: $R e=500, s=-1$, Stewartson branch. Right: $R e=500, s=0.5$, Batchelor branch. The fast dynamo (left) is associated with inwards energy flux. The slow dynamo (right) has outwards energy flux for some $R e_{m}$. The field is normalised so that $T(1)=1$, while $\lambda<0$ is plotted as $\lambda=0$.
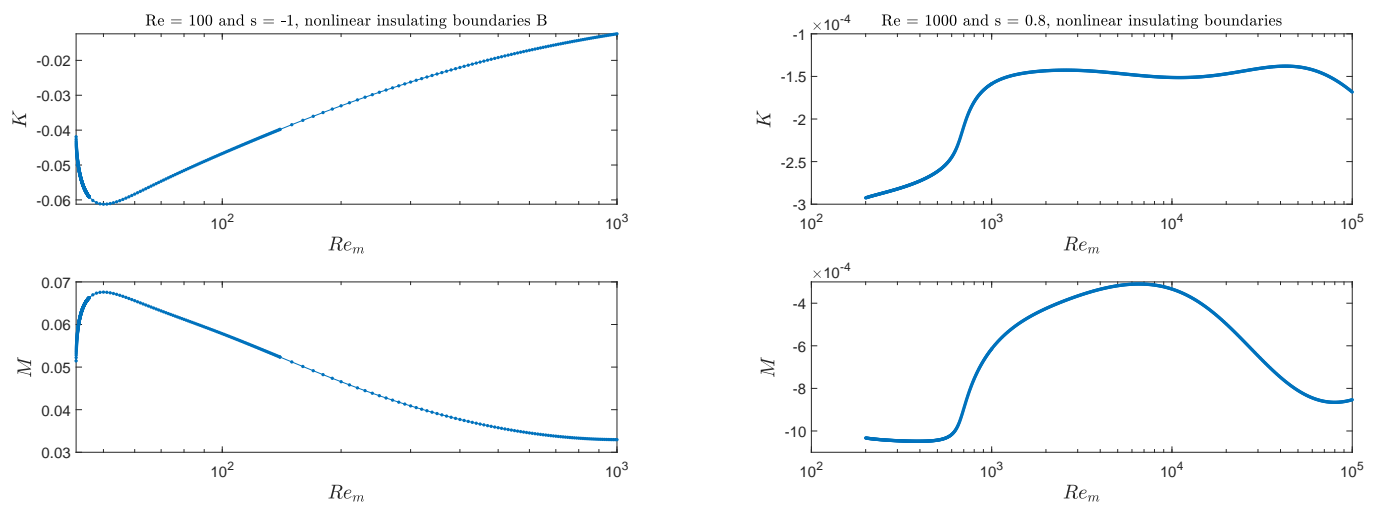

Figure 12. Kinetic and magnetic energy fluxes at large $r$ for nonlinear equilibria. Left: insulating discs, $R e=100, s=-1$, asymmetric solution branch. Kinetic flux is outwards, but the magnetic flux is inwards. Right: insulating discs $R e=1000, s=0.8$, Both the kinetic and magnetic fluxes are outwards.

does not hold for relatively small variations from axisymmetry at high $R e_{m}$. In general, it is physically likely that the local mechanism for field generation will overcome the distant Joule dissipation, for high enough $R e_{m}$.

\section{Concluding remarks}

It has long been recognised that differentially rotating flows are good candidates for dynamos, and the VKS experiment (Nore et al. 2018, Berhanu et al. 2010, Monchaux et al. 2009) has demonstrated that these are realisable in the laboratory, at least for soft-iron rotors. We have shown how a simple, laminar flow can to some extent describe such dynamos, even into the nonlinear regime. In reality, the flow would be turbulent, but we would hope that at least qualitative features of the laminar description would apply. The finite extent of experimental rotors also limits the practical applicability of our model.

Ferromagnetic boundaries are found to be conducive to dynamo action. We find the laminar dynamos with fastest growth occur for high Re and for counter-rotating discs. These are advantageous not just because of the increased shear, but also because the induced poloidal flow is usually directed towards each disc, which supports a fast dynamo. However, such flows tend to be associated with an inward magnetic energy flux at infinity and so may not create a functioning dynamo when the system is embedded in a finite container. Our study predicts that for dynamo action the average rotation rate of the two discs should ideally be non-zero to enable swirl between the discs.

Our dynamos saturate in a steady state. As $R e_{m} \rightarrow \infty$ at constant but large $R e$, for perfectly conducting walls, the magnetic activity is quenched apart from a uniform current within the fluid. For insulating walls, 
however, an asymptotic state is approached in which the dynamo action localises about one or other of the discs, even for the exactly symmetric configuration. Admittedly, this limit corresponds to a high magnetic Prandtl number and so is of questionable physical relevance.

The laminar model between infinite discs is surprisingly simple. The most unstable mode may have a different spatial structure to that we have assumed, and may well not be even approximately axisymmetric. The critical $R e_{m}$ we have found must therefore be interpreted as an upper bound. For the kinematic problem, it would be possible to consider fields $\propto \mathrm{e}^{\mathrm{i} m \theta}$. However, a simple radial structure would only apply in the high $R_{m}$ limit. Other azimuthal modes would naturally be excited in the nonlinear case.

The greatest limitation on our model will derive from the behaviour at large $r$ for finite rotors, when apart from anything else Cowling's theorem may apply unless appropriate symmetry-breaking steps are taken. We have shown that in some parameter ranges magnetic energy is being drawn in from infinity, and these solutions cannot be considered dynamos in the usual sense. However, in many cases the magnetic energy flux is outwards and these local solutions could well drive a full nonlinear dynamo.

There are parameter ranges with more than one hydrodynamic solution where one is associated with outward magnetic energy flux and the other inward. In a finite domain, we have argued that the first may give rise to a dynamo when the second does not. If that is true, it is conceivable that the dynamo could act as a switch. An initial flow could give rise to a dynamo which reacted back on the flow knocking it into a state which quenched the magnetic field entirely, leaving a different hydrodynamic state. This is all speculative, as we have not investigated time-dependent flows in the nonlinear regime, while the precise conditions at large $r$ will be important.

\section{REFERENCES}

Batchelor, G., Note on a class of solutions of the NavierStokes equations representing steady rotationallysymmetric flow. Quart. J. Mech. Appl. Math, 1951, 4, 29-41.

Berhanu, M., Verhille, G., Boisson, J., Gallet, B., Gissinger, C., Fauve, S., Mordant, N., Ptrlis, F., Bourgoin, M., Odier, P., Pinton, J.F., Plihon, N., Aumatre, S., Chiffaudel, A., Daviaud, F., Dubrulle, B. and Pirat, C., Dynamo regimes and transitions in the VKS experiment. Eur. Phys. J. B, 2010, 77, 459468.

Cowling, T.G., The Magnetic Field of Sunspots. MNRAS, 1933, 94, 39-48.

Gailitis, A., Lielausis, O., Platacis, E., Dementev, S., Cifersons, A., Gerbeth, G., Gundrum, T., Stefani, F., Christen, M. and Will, G., Magnetic eld saturation in the Riga dynamo experiment. Phys. Rev. Lett., 2001, 86, 30243027.

Hewitt, R. and Al-Azhari, M., Non-axisymmetric self-similar flow between two rotating disks. J. Eng. Math., 2009, 63, 259-277.

Ledoux, V., Daele, M.V. and Berghe, G.V., MATSLISE: A MATLAB package for the numerical solution of Sturm-Liouville and Schrödinger equations. ACM Transactions on Mathematical Software (TOMS), 2005, 31, 532-554.

Monchaux, R., Berhanu, M., Aumatre, S., Chiffaudel, A., Daviaud, F. Dubrulle, B., Ravelet, F., Fauve, S., Mordant, N., Ptrlis, F., Bourgoin, M., Odier, P., Pinton, J.F., Plihon, N. and Volk, R., The von Kármán Sodium experiment: Turbulent dynamical dynamos. Phys. Fluids, 2009, 21.

Nore, C., Castanon Quiroz, D., Cappanera, L. and Guermond, J.L., Numerical simulation of the von Kármán sodium dynamo experiment. J. Fluid Mech., 2018, 854, 164-195.

Roberts, P.H. and Soward, A.M., Dynamo theory. Annu. Rev. Fluid Mech., 1992, 24, 459-512.

Shampine, L.F., Gladwell, I., Shampine, L. and Thompson, S., Solving ODEs with matlab, 2003 (Cambridge university press).

Stewartson, K., On the flow between two rotating coaxial discs. Math. Proc. Cam. Phil. Soc., 1953, 49, $333-341$.

Stieglitz, R. and Mller, U., Experimental demonstration of a homogeneous two-scale dynamo. Phys. Fluids, 2001, 13, 561564.

Vaz, R.H., Boshier, F. A, T. and Mestel, A.J., Dynamos in an annulus with fields linear in the axial coordinate. Geophys. Astrophys. Fluid Dyn., 2018, 112, 222-234.

Von Kármán, T., ber laminare und turbulente Reibung. Z. angew. Math. Mech., 1921, p. 233252. 
